\title{
Half-Metallicity in Undoped and Boron Doped Graphene Nanoribbons in Presence of Semi-local Exchange-Correlation Interactions
}

\author{
Sudipta Dutta and Swapan K. Pati* \\ Theoretical Sciences Unit and DST unit on Nanoscience \\ Jawaharlal Nehru Centre For Advanced Scientific Research \\ Jakkur Campus, Bangalore 560064, India.
}

\begin{abstract}
We perform density functional calculations on one-dimensional zigzag edge graphene nano-ribbons (ZGNRs) of different widths, with and without edge doping including semi-local exchange-correlations. Our study reveals that, although the ground state of edge passivated (with hydrogen) ZGNRs prefers to be anti-ferromagnetic, the doping of both the edges with Boron atoms stabilizes the system in a ferromagnetic ground state. Both the local and semi-local exchange-correlations result in half-metallicity in edge passivated ZGNRs at a finite cross-ribbon electric field. However, the ZGNR with Boron edges shows half-metallic behavior irrespective of the ribbonwidth even in absence of electric field and this property sustains for any field strength, opening a huge possibility of applications in spintronics.
\end{abstract}


Nanomaterials of Carbon of different dimensionalities like Carbon nanotubes, fullerenes etc. have been a subject of interest over the past few decades due to their potential applications in various nanoscale electronic devices [1, 2, 3]. Graphene, the two dimensional flat monolayer of Carbon atoms packed into a honeycomb lattice is the latest addition to this family owing to the recent progress in experimental techniques [4, 5, 6, 7]. Because of its sophisticated low-dimensional electronic properties and huge application possibility, it has attracted the focus of a big scientific community in recent times to explore it in various aspects [8, 9, 10, 11, 12, 13, 14, 15].

The electronic properties of nanoscale Carbon systems are governed by their size and geometry. Recent progress in experiments allows to make finite size graphene layer, termed as graphene nanoribbons (GNRs) with varying widths, either by cutting mechanically exfoliated graphenes [4, 5, 6] and patterning by electron beam lithography[16], or by controlling the epitaxial growth of graphenes[17, 18]. Different possibilities of geometrical termination of graphene layer give rise to two different edge geometries, namely, zigzag and armchair edges, differing largely in their electronic properties. These different edge geometries have been modeled by imposing different boundary conditions on Schrodinger's equation within the tight-binding limit [19, 20, 21, 22] or on the Dirac equation for two-dimensional massless fermions with an effective speed of light [23, 24, 25, 26] in previous studies. There exists a few many body studies also on the low-dimensional electronic properties [27, 28, 29, 30, 31] of these systems. These also have been exten- 
sively studied using density functional theory [32, 33].

One-dimensional antiferromagnetic zigzag edge graphene nano-ribbons (ZGNRs) show half-metallicity at a finite external electric field across the ribbon width within local density approximation (LDA) as observed by Son et. al. 32]. Half-metallic materials show zero band gap for electrons with one spin orientation and semiconducting or insulating band gap for the other, giving rise to completely spin polarized current, which has already been observed in some materials like Heusler compounds 34], manganese perovskite 35] etc. However, one recent study predicted that, in presence of non-local exchangecorrelations like B3LYP, the cross-ribbon electric field makes the band gap of one spin orientation insulating and the other semiconducting for a finite size edge passivated ZGNR with possible application as spin-selective semiconductors 36].

To enlighten the controversy on the half-metallicity of ZGNRs in presence of non-local exchange-correlations, we study 1D periodic ZGNRs of different widths with a variety of edge passivation and doping using $a b$ initio density functional package, SIESTA[37]. We have performed spin polarized calculations within generalized gradient approximation (GGA) considering Perdew-Burke-Ernzerhof (PBE) exchange and correlation functionals 38 ] with double zeta polarized (DZP) basis set. GGA approximation takes into account the semi-local exchange-correlations which have significant impact on low-dimensional spin systems like GNRs. The 1D ZGNRs along $x$-axis and in $x y$-plane are represented by the unit cell lattice vectors $2.45,30.0,16.0$, 


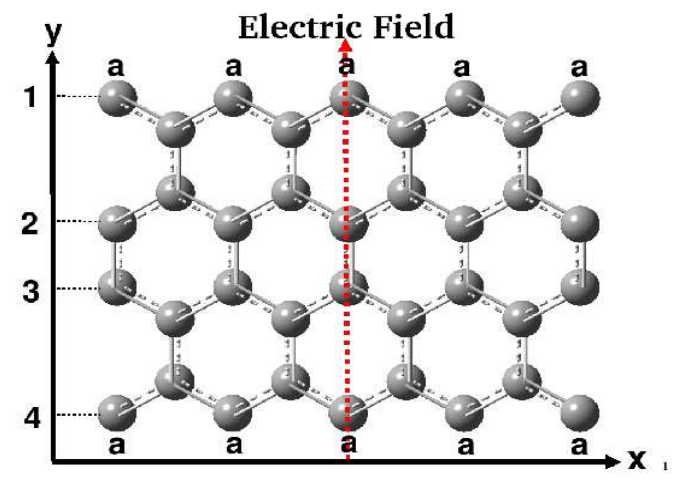

Figure 1: (Color online) Model system: a zigzag edge 1D graphene with 4 zigzag chains (4-ZGNR), translated along $x$-axis. The edge atoms are denoted by "a". The dotted arrow shows the direction of external electric field.

which create sufficient vacuum, ensuring no interaction between the adjacent layers [32]. We have considered 400 Rydbergs energy cut-off for a real space mesh size and a k-point sampling of $36 \mathrm{k}$-points, uniformly positioned along the 1D Brillouin zone. The systems considered are depicted in Fig.1 with (i) all the edge Carbon atoms passivated by hydrogen and (ii) all the edge Carbons replaced (doped) with Boron (B). We vary the ribbon width $(w)$ for both the cases from 4 to 10 zigzag chains, and relax all the structures for both ferromagnetic and antiferoomagnetic spin orientations. We then apply a transverse electric field along the cross-ribbon direction, i.e., along the $y$ axis as shown in Fig.1 and track the band gap variation for both the spins orientations.

The undoped ZGNRs with hydrogen-passivated edges show that the antiferromagnetic ground state is stabler than the ferromagnetic state by a 


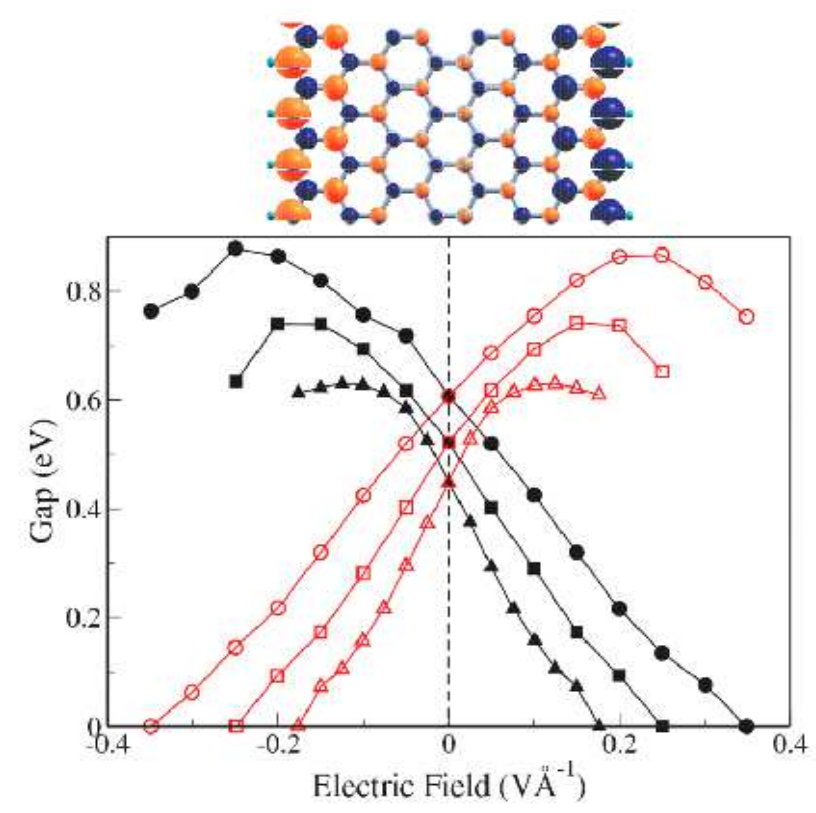

Figure 2: (Color online) Top picture shows the accumulation of up spin and down spin densities on either edges of a finite segment of 1D 8-ZGNR with hydrogen passivated edges in absence of electric field. The down panel shows the up spin (closed symbols) and down spin (open symbols) gaps for 6 (circle), 8 (square) and 10-ZGNRs (triangle) as a function of electric field. Negative field corresponds to reverse direction. 
small margin, of the order of meV. The ground states with zero magnetic moment show antiparallel alignment of spins at opposite edges as well as between nearest neighbors, as depicted in Fig.2. These observations are consistent with the previous LDA results 32 as well as with the theory based on the Hubbard Hamiltonian for bipartite lattice 39]. However, the electronelectron interactions can overcome such a small gap to make the ground state of ZGNRs ferromagnetic [27]. In absence of external electric field, both the upspin and downspin band gaps show insulating behavior and the insulating gap decreases with the increase in width, as can be seen from Fig.2. The gap appears to be more than the previously reported LDA gap, since LDA is known to underestimate the band gap. The external electric field perturbs the ground state band structure. Increase in field strength along positive direction decreases the band gap for the upspin and at a finite electric field strength, the up-spin channel becomes metallic. The band gap for downspin however, increases with the field strength. As a result, the 1D ZGNRs become half-metallic beyond a finite critical field strength, termed as $E_{c, w}$. From Fig.2 it is clear that, the $E_{c, w}$ decreases with the increase in width $(w)$. Electric field in reverse direction closes the down-spin gap and increases the up-spin insulating gap. Thus, different directions of external electric field open up different spin channels. Since, the edges have different spin polarizations, the applied bias $e E y(e, E$ and $y$ are electronic charge, electric field and the distance respectively) in either directions affects the up-spin and down-spin band gaps in opposite fashions. Our results thus suggest that 
in presence of gradient corrected semi-local exchange-correlations, the 1D ZGNRs do behave as half-metallic materials beyond a certain electric field strength. This result strongly contradicts the observation of spin-selected semiconducting behavior of ZGNRs in presence of non-local exchange- correlation as found by Rudberg et al[36]. The controversy of semiconducting gap for one spin channel arises due to the finite size of the system, used in ref[36] and such a gap can be tuned with increase in length (along $x$-axis) of ZGNRs. The gradual spin density annihilation at the edges of finite size ZGNRs with increase in electric field as observed by Rudberg et al[36] in presence of non-local exchange-correlation is nevertheless due to finite size effect [40]. Moreover, very high field strength as considered in ref[36] is unphysical (beyond perturbing regime) and leads to collapse of the structure of such a nano-scale system.

The alternate idea of edge passivation is to dope the edge atoms by three coordinated Boron atoms, with introduction of holes in the system. We find that $\mathrm{B}$ doping at the edges makes the ground state of ZGNRs ferromagnetic. Such a possibility with large hole doping in strongly correlated low-dimensional systems was proposed earlier [41]. In our case, the energy difference between the ferromagnetic and antiferromagnetic ground state is of the order of meV. In the ground state, the electrons at the same edges as well as at the opposite edges are parallely aligned (see Fig.3). In Fig.4, we plot the density of states (DOS) for this system for zero and nonzero electric field. As can be seen, even in absence of any external electric field, this 


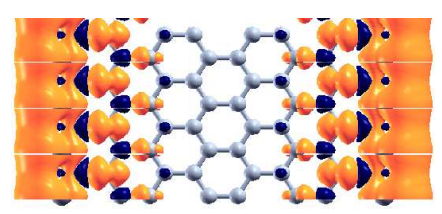

Figure 3: (Color online) Accumulation of up spin densities on both the edges of a finite segment of 1D 8-ZGNR with all the edge atoms doped with Boron in absence of electric field.

system shows metallic behavior for the majority spin and insulating behavior for the minority spin, the typical half-metallic behavior. The increase in field strength in either directions does not change this behavior: the majority spin channel always remains conducting and the minority spin gap remains insulating with almost same gap for all the field strengths (see Fig.4). It is because, the external field in either directions affects both the spins channels similarly, since the spin polarization of the opposite edges are same in this system. As a consequence, the change in direction of the external field cannot change the spin polarization of the current. The minority spin gaps for all the widths are much higher compared to the room temperature $(0.025 \mathrm{eV})$. Thus, the ZGNRs with both the edges doped with B can act as a potential half-metallic material for any external electric field over a large temperature domain irrespective of its width. The projected DOS (pDOS) analysis shows that, the half metallicity at Fermi energy in this system is largely induced by the B orbitals at the edges.

We have also studied the ZGNRs of several widths with both the edges doped by Nitrogen (N) atoms. This system shows antiferromagnetic ground 


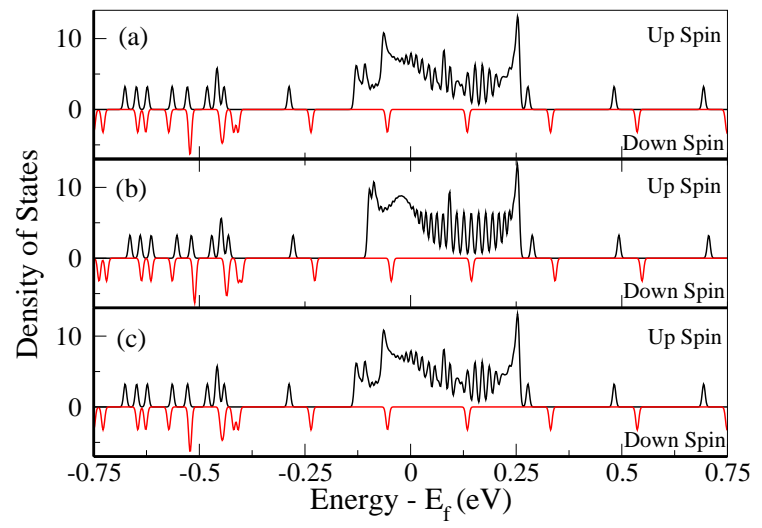

Figure 4: (Color online) Up and down spin density of states of 8-ZGNR with both the edges doped by Boron as a function of energy, scaled with respect to the Fermi energy for three different electric fields $(a)-0.2,(b) 0.0$ and (c) $0.2 V \stackrel{\circ}{A}^{-1}$.

state with different spin polarization of the opposite edges. However, although the electron accumulation on the edges is noticably low compared to the systems discussed above, this system shows metallic behavior for both the spins at Fermi energy even in absence of external electric field. The pDOS analysis shows that, the $\mathrm{N}$ orbitals contribute largely to the DOS at Fermi energy making the system metallic. All these observations remain consistent with the increase in width.

In summary, we have studied the 1D ZGNRs of different widths with edge passivation and with edge doping in presence of transverse electric field. Our study shows that, even in presence of gradient corrected semi-local exchangecorrelations, the edge passivated 1D ZGNRs can act as half-metallic materials beyond a certain critical field strength, which decreases with the increase in 
ribbon width. However, Boron doped ZGNRs show half-metallic behavior even in absence of external electric field and this half-metallicity sustains for any finite field strength over a large temperature range. This observation opens a huge possibility of application of B doped ZGNRs in spintronic devices.

Acknowledgement: SD acknowledges the CSIR for the research fellowship and SKP acknowledges the research support from DST and CSIR, Govt. of India.

\section{References}

[1] Dresselhaus, G.; Dresselhaus M. S.; Eklund, P. C. Science of Fullerenes and Carbon Nanotubes: Their Properties and Applications (Academic, New York, 1996).

[2] Chico, L.; Crespi, V. H.; Benedict, L. X.; Louie, S. G.; Cohen, M. L. Phys. Rev. Lett. 1996, 76, 971.

[3] McEuen, P. L.; Fuhrer, M. S.; Park, H. IEEE Trans. Nanotechnol. 2002, 1,78 .

[4] Novoselov, K. S. et. al. Proc. Natl. Acad. Sci. U. S. A. 2005, 102, 10451.

[5] Novoselov, K. S. et. al. Nature (London) 2005, 438, 197.

[6] Zhang, Y.; Tan, Y. W.; Stormer, H. L.; Kim, P. Nature (London) 2005, 438, 201. 
[7] Stankovich, S. et. al. Nature (London) 2006, 442, 282.

[8] Meyer, J. C. et. al. Nature 2007, 446, 60.

[9] Novoselov, K. S. et. al. Science 2007, 315, 1379.

[10] Geim, A. K.; Novoselov, K. S. Nature Materials 2007, 6, 183.

[11] Katsnelson, M. I. Mater Today 2007, 10, 20.

[12] Schniepp, H. C. et. al. J. Phys. Chem. B 2006, 110, 8535.

[13] Jiang, D.; Sumpter, B. G.; Dai, S.; J. Phys Chem. B 2006, 110, 23628.

[14] Agapito, L. A.; Cheng, H-P. J. Phys. Chem. C 2007, 111, 14266.

[15] Grimme, S.; Muck-Lichtenfeld, C.; Antony, J. J. Phys. Chem. C 2007, $111,11199$.

[16] Ozyilmaz, B. et. al. Phys. Rev. Lett. 2007, 99, 166804.

[17] Berger, C. et. al. Science 2006, 312, 1191.

[18] Berger, C. et al. J. Phys. Chem. B 2004, 108, 19912.

[19] Fujita, M.; Wakabayashi, K.; Nakada, K.; Kusakabe, K. J. Phys. Soc. Jpn. 1996, 65, 1920.

[20] Nakada, K.; Fujita, M.; Dresselhaus, G.; Dresselhaus, M. S. Phys. Rev. $B$ 1996, 54, 17954 . 
[21] Wakabayashi, K.; Fujita, M.; Ajiki, H.; Sigrist, M. Phys. Rev. B 1999, $59,8271$.

[22] Ezawa, M. Phys. Rev. B 2006, 73, 045432.

[23] Brey, L.; Fertig, H. A. Phys. Rev. B 2006, 73, 235411.

[24] Sasaki, K. I.; Murakami, S.; Saito, R. J. Phys. Soc. Jpn. 2006, 75, 074713 .

[25] Abanin, D.A.; Lee, P. A.; Levitov, L. S. Phys. Rev. Lett. 2006, 96, 176803.

[26] Castro Neto, A. H.; Guinea, F.; Peres N. M. R. Phys. Rev. B 2006, 73, 205408.

[27] Dutta, S.; Lakshmi, S.; Pati, S. K. cond-mat/0706.2528v1 2007.

[28] McCann, E. et. al. Phys Rev. Lett. 2006, 97, 146805.

[29] Yang, L.; Cohen, M. L.; Louie, S. G. Nano Letters 2007, 7, 3112.

[30] Prezzi, D.; Varsano, D.; Ruini, A.; Marini, A.; Molinari, E. cond-mat/07060916 2007.

[31] Hikihara, T.; Hu, X.; Lin, H-H.; Mou, C-Y. Phys. Rev. B 2003, 68, 035432. 
[32] Son, Y. W.; Cohen, M. L.; Louie, S. G. Nature (London) 2006, 444, 347.; Son, Y. W.; Cohen, M. L.; Louie, S. G. Phys. Rev. Lett. 2006, 97, 216803.

[33] Okada, S.; Oshiyama, A. Phys. Rev. Lett. 2001, 87, 146803.

[34] de Groot, R. A.; Mueller, F. M.; van Engen, P. G.; Buschow, K. H. J. Phys. Rev. Lett. 1983, 50, 2024.

[35] Park, J-H. et. al. Nature 1998, 392, 794.

[36] Rudberg, E.; Salek, P.; Luo, Y. Nano Letters 2007, 7, 2211.

[37] Soler, J. M. et. al. J. Phys. Condens. Matter 2002, 14, 2745.

[38] Burke, K.; Perdew, J. P.; Ernzerhof, M. Int. J. Quantum Chem. 1997, $61,287$.

[39] Lieb, E. H. Phys. Rev. Lett. 1989, 62, 1201.

[40] Kan, E-J.; Li, Z.; Yang, J.; Hou, J. G. cond-mat/0708.1213v12007.

[41] Shastry, B. S.; Krishnamurthy, H. R.; Anderson, P. W. Phys. Rev. B 1990, 41, 2375. 